\title{
Acute Changes in Hamstrings Musculo-Articular Dissipative Properties Induced by Cyclic and Static Stretching
}

\author{
Key words \\ passive torque \\ - hysteresis \\ potential elastic energy \\ viscosity \\ thixotropy
}

\author{
A. Nordez ${ }^{1}$, P . M c N a i r ${ }^{2}$, P. Casari ${ }^{3}$, C. Cornu ${ }^{1}$ \\ ${ }^{1}$ Université de Nantes, Nantes Atlantique Universités, Laboratoire “Motricité, \\ Interactions, Performance”, JE 2438, UFR STAPS, Nantes, France \\ 2 Physical Rehabilitation Research Centre, Auckland University of Technology, \\ Auckland, New Zealand \\ 3 Université de Nantes, Nantes Atlantique Universités, CNRS, Institut de \\ Recherche en Génie Civil et Mécanique, UMR 6183, UFR des Sciences et \\ Techniques, Nantes, France
}

\begin{abstract}
This study was designed to measure changes in musculo-articular dissipative properties related to viscosity that were induced by passive cyclic and static stretching. Musculo-articular dissipative properties were assessed by calculating a dissipation coefficient using potential elastic energies stored and restituted during cyclic stretching. Eight subjects performed five passive knee extensions/flexions cycles on a Biodex ${ }^{\circledR}$ dynamometer at $5^{\circ} \cdot \mathrm{s}^{-1}$ to $80 \%$ of their maximal range of motion before and after a static stretching protocol. Electromyographic activity from the hamstring muscles was monitored and remained constant during cyclic stretching and after static
\end{abstract}

\section{Introduction}

Passive stretching exercises are commonly performed in sports and rehabilitation. Both animal [20] and human data $[6,9-11,14,15]$ have indicated that the biomechanical properties (e.g., force, energy, stiffness) of passive muscle are altered immediately after passive stretching. In humans, the passive mechanical properties of a musculo-articular complex, including structures spanning the joint (i.e., muscles, tendons, skin, subcutaneous tissue, fascia, ligaments, joint capsule and cartilage) [18], can be assessed using passive loading and unloading torque $(\mathrm{T})$ - angle $(\theta)$ responses $[6,9-11,14,15,18]$. From these data, potential elastic energy stored by the musculoarticular complex during loading can be determined by calculating the area under the loading $\mathrm{T}-\theta$ relationship. It has already been shown that the potential elastic energy stored, stiffness and the maximal passive torque during loading are decreased by stretching $[9,11,14,15]$, and that this effect is temporary [11]. stretching $(\mathrm{p}>0.05)$. The dissipation coefficient decreased during cyclic stretching $(-28.8 \pm 6.0 \%$, $\mathrm{p}<0.001)$, while it was slightly increased after static stretching $(+3.8 \pm 5.0 \%, p=0.037)$. The findings showed that energy stored and energy restituted decreased during cyclic stretching and after static stretching $(\mathrm{p}<0.05)$. During unloading, passive torque remained constant during cyclic stretching, but was decreased after static stretching. The findings indicate that musculoarticular dissipative properties were primarily affected by a single cycle of motion, and were not influenced by static stretching procedures. The decrease in dissipation coefficient following cyclic motion indicates that the musculo-articular system displays thixotropic behavior.

Potential elastic energy stored by a musculo-articular complex during loading is not completely restituted during unloading leading to a hysteresis effect (see $\bigcirc$ Fig. 1). Three physical phenomena could explain the dissipation of potential elastic energy during loading and unloading at a constant velocity: friction, plasticity, and viscosity $[12,16]$. The role of friction is thought to be quite limited [5], and since plasticity implies irreversible alterations, authors have considered that the isolated muscle-tendon unit [20] and the in vivo musculo-articular complex $[5,6,11,15]$ exhibit viscoelastic behavior. Consequently, the dissipation of energy by the musculo-articular complex could be interpreted as an indication of viscosity. The dissipative properties of such a system can be quantified using a dissipation coefficient (DC, equation [1]) corresponding to the normalized area of the hysteresis (see $\bullet$ Fig. 1) [12, 16]. Though this measure has not been extensively used previously in in vivo research, this coefficient may be valuable in that it enables one to quantify capacities of energy storage and restitution of the musculo-articular complex. To our 

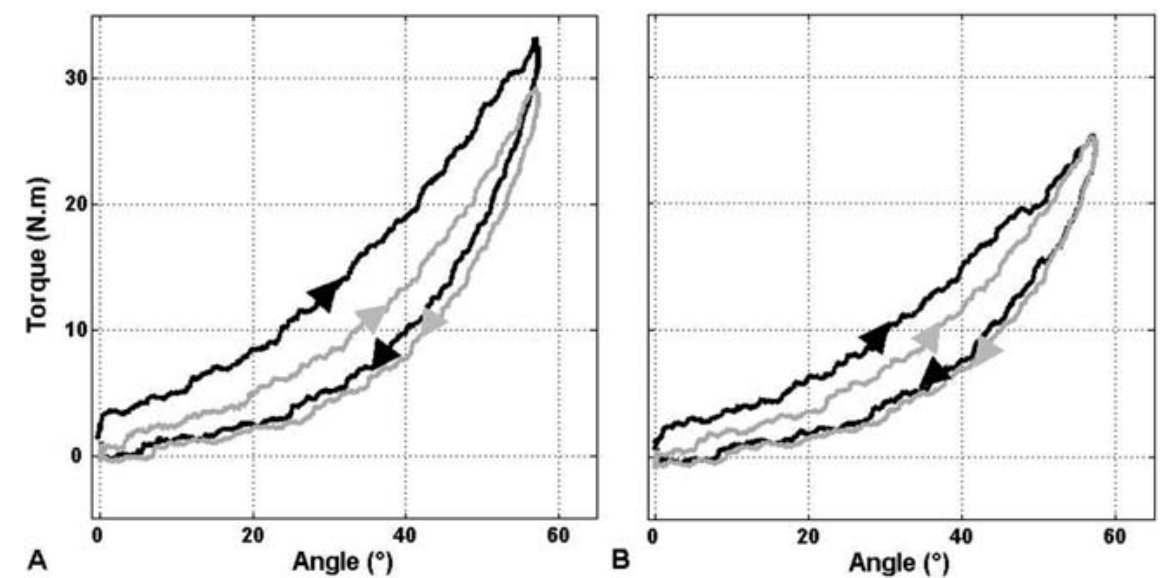

Fig. 1 A and $\mathbf{B}$ Typical load and unload passive torque-angle relationships $\mathbf{A}$ before static stretching B after static stretching. - first cycle, - fifth cycle. Energy stored and restituted can be assessed as the area under loading and unloading curves respectively. The hysteresis area is calculated for each cycle as the area between load and unload curves (i.e., energy stored minus energy restituted), and the dissipation coefficient is the area of hysteresis divided by the area under the load curve. knowledge, only Magnusson et al. [11] have studied viscous changes in vivo during passive motion. They found that the hysteresis area was decreased during cyclic stretching.

Decreases in viscosity may affect the shock absorbing ability of the musculotendinous units. It may be that muscles with less shock absorbing ability are more prone to injury, or cannot protect joint structures such as the articular cartilage as effectively. No previous work has compared the effects of cyclic and static stretching. Yet, these types of stretches are typically used in sports training and rehabilitation. It may be that one type of stretching induces greater changes to dissipative properties. Previous research by McNair et al. [14] has shown that related elastic parameters such as stiffness are more affected by cyclic motion compared to static stretching. However, these authors did not examine dissipative properties.

Therefore, in respect to viscous changes that occur with stretching activities in vivo, there is a lack of fundamental information that would be important in understanding the mechanical basis to stretching. The objective of this study was to compare the viscous changes that occur with static and cyclic stretching by quantifying the energy absorbed and dissipated during passive knee joint extension and flexion.

\section{Materials and Methods}

\section{Subjects}

Eight healthy males $(23.3 \pm 1.9$ years, height $181.3 \pm 7.0$ $\mathrm{cm}$, weight $74.3 \pm 4.7 \mathrm{~kg}$ ) volunteered to participate in this study and signed an informed consent form. All procedures used in this study were in conformity with the Declaration of Helsinki. Subjects practiced recreational sports, but did not participate in any strength or flexibility training at the time of the study. No subjects had sustained a recent injury that may have affected the findings.

\section{Measurement techniques}

The Biodex system 3 research ${ }^{\circledR}$ isokinetic dynamometer (Biodex medical, Shirley, NY, USA) was used to measure torque produced in resistance to passive stretch $(T)$, knee joint angle $(\theta)$ and knee joint angular velocity $(\omega)$. The measurement method was similar to that used by other investigators [9-11]. Subjects were seated and the thigh was fastened using Velcro straps to a specially constructed thigh pad elevating it from horizontal and also minimizing contact between electromyographic electrodes and the seat surface. The trunk - thigh angle was adjusted at $60^{\circ}$, and the input axis of the dynamometer was aligned with the approximate axis of rotation of the knee joint. All subjects were unable to reach full knee extension in this position. All procedures began with the lower leg perpendicular to the thigh. This position was used to determine our reference knee angle expressed as $0^{\circ}$. Surface electromyographic signals (sEMG) of the hamstring muscles were recorded in order to ensure that no undesirable activation occurred during the stretches. Bipolar surface electrodes (Ag - AgCl, 4 mm diameter, In Vivo Metric, Healdsburg, CA, USA) were placed over the semitendinosus (ST) and biceps femoris $(\mathrm{BF})$ muscles with a $13 \mathrm{~mm}$ inter-electrode distance. Electrode-skin impedance was reduced below $55 \mathrm{k} \Omega$ using standard skin preparation procedures, and surface electrodes were placed between the distal tendon and the innervation zone with respect to the assumed fibers' direction according to the Surface Electromyography for the Non-Invasive Assessment of Muscles (SENIAM) recommendations [7]. Two reference electrodes were placed over lateral and medial epicondyles. Mechanical signals (T, $\theta$ and $\omega$ ) and sEMG signals were sampled at $256 \mathrm{~Hz}$ and $1024 \mathrm{~Hz}$, respectively, with a 12 bits A/D converter (Myodata, Electronique du Mazet, France). Data were stored in a flash memory card (20 Mo) and transferred to a computer hard disk for further analysis.

\section{Experimental protocol}

All subjects performed two sessions with a one-day rest period in-between. First, a familiarization session was performed in order to prepare the subjects for all testing procedures. During the main session, a baseline test was followed by a static stretching protocol, and thereafter a posttest was performed. To limit the effects of the baseline test, a 15-min rest period was observed before the static stretching protocol. No rest period was observed between the static stretching protocol being completed and the posttest. Baseline and posttests were similar and included the following: i) a maximal knee range of motion (ROM) measurement. In this test, the lower leg was passively extended $\left(\omega=5^{\circ} \cdot \mathrm{s}^{-1}\right)$, and the subjects used a stop switch when they perceived the maximum tolerable hamstring muscle stretch. This point was operationally defined as their maximal range of motion (ROM). The leg was then immediately returned to the starting position; ii) five cyclic $\left(\omega=5^{\circ} \cdot \mathrm{s}^{-1}\right)$ passive repetitions at $80 \%$ of the ROM measured during the baseline test; and iii) three maximal isokinetic knee flexions and extensions at $60^{\circ} \cdot \mathrm{s}^{-1}$ were 


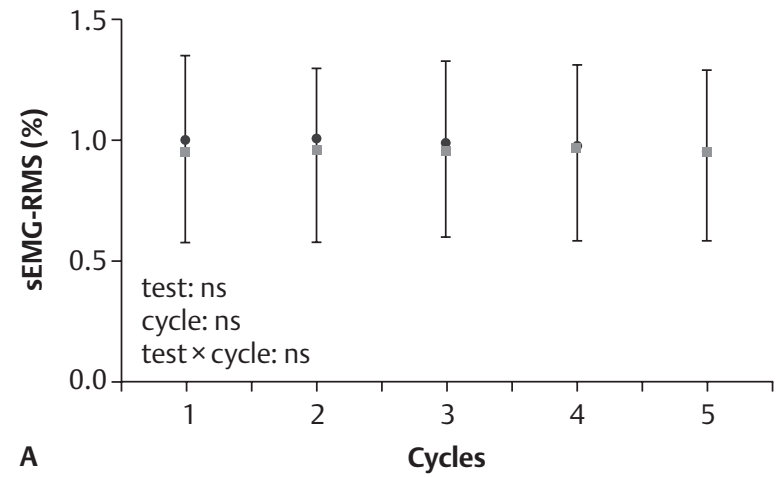

Fig. 2 A and $\mathbf{B}$ Changes in $\mathbf{A}$ averaged normalized root mean square of surface electromyographic signals (sEMG-RMS) of semitendinosus and biceps femoris muscles during cyclic stretches, and $\mathbf{B}$ dissipation coefficient (DC)

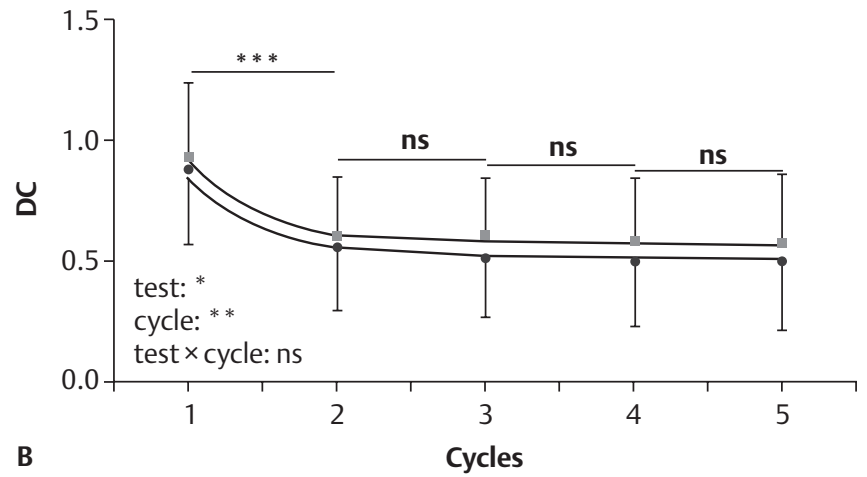

before - and after $\square$ static stretching. ns: nonsignificant, ${ }^{*} p<0.05$, ${ }^{* *} \mathrm{p}<0.01,{ }^{* * *} \mathrm{p}<0.001$ performed after the posttest in order to normalize the sEMG data.

The stretching protocol was composed of six 30 s static hamstring stretches. Specifically, the leg was passively extended to a predetermined knee angle $\left(\omega=5^{\circ} \cdot \mathrm{s}^{-1}\right)$, maintained for $30 \mathrm{sec}$ onds in this position and unloaded to the initial position $\left(\omega=5^{\circ} \cdot \mathrm{s}^{-1}\right)$. The predetermined knee angle was $80 \%$ of the pretest ROM for the first stretching repetition. It was then increased with a $2 \%$ step between each repetition until $90 \%$ of the ROM for the sixth repetition. No rest period was provided between each stretching repetition.

\section{Data analysis}

All the data were processed using a standardized program computed with Matlab ${ }^{\circledR}$ (The Mathworks, Natick, MA, USA). First, mechanical signals (T, $\theta$ and $\omega$ ) and sEMG signals were filtered using Butterworth second order low pass filter $(10 \mathrm{~Hz})$ and Butterworth second order band pass filter $(6-400 \mathrm{~Hz})$, respectively. Next, recorded torque was gravity corrected [1]. Thereafter, the energy stored during loading (E, i.e., the area under load curve, see $\odot$ Fig. 1) the energy restituted (ER, i.e., the area under unload curve, see $\bigcirc$ Fig. 1) and the energy dissipated by the musculo-articular complex (ED, i.e., the hysteresis area, see $\bullet$ Fig. 1) were calculated. ED was divided by the $\mathrm{E}$ to calculate the dissipation coefficient (DC, equation 1 ).

$\mathrm{DC}=\frac{\mathrm{ED}}{\mathrm{E}}=\frac{\mathrm{E}-\mathrm{ER}}{\mathrm{E}}=1-\frac{\mathrm{ER}}{\mathrm{E}}$

One dimensional interpolations using the discrete Fourier transform method were used to assess passive torque every $2 \%$ of the ROM for each loading and unloading curves. Averaged root mean squares of sEMG signals (sEMG-RMS) of ST and BF muscles were calculated during the passive knee extensions. Passive sEMG-RMS were normalized to the sEMG-RMS calculated during the isokinetic phase of motion of maximal effort knee flexion at $60^{\circ} \cdot \mathrm{s}^{-1}$ performed after the posttest.

After checking the distribution of data (Kolmogorov-Smirnov test), parametric statistical tests were performed using Statisti$\mathrm{ca}^{\circledR}$ software (StatSoft, Tulsa, OK, USA). A paired $t$-test was done to compare the ROM between baseline and posttests. The only way to examine the effects of stretching on the DC was to use the cyclic stretching repetitions. However, it was also of interest to examine the DC across repetitions of cyclic motion. Thus, the statistical analyses of DC and normalized sEMG-RMS involved two separates $2 \times 5$ ("test" × "cycle") repeated measures analyses of variance (ANOVAs). Where significant differences were found, $E$ and ER were analyzed in order to appreciate their influence on the DC. Changes in E and ER involved two separate two-way repeated measures ANOVAs ("test" × "cycle"). Newman-Keuls post hoc analyses were used when appropriate. The critical level of significance in the present study was set at $\mathrm{p}<0.05$. Data are expressed as means \pm standard deviations.

\section{Results}

Range of motion

The averaged ROM which was $78.8 \pm 6.4^{\circ}$ at baseline was signifi-cantly increased after static stretching $(+7.5 \pm 6.5 \%, \mathrm{p}<$ $0.01)$.

\section{sEMG activity}

sEMG activity was not changed across cyclic stretches nor after static stretching with a mean and standard deviation of $0.97 \pm$ $0.33 \%$ (range $0.49-1.57 \%$, $\odot$ Fig. $2 \mathrm{~A}$ ).

\section{Dissipation coefficient}

Significant main effects were seen for "test" ( $p<0.05$ ) and "cycle" $(p<0.001)$, while no interaction test $\times$ cycle was noted $(p>0.05)$ ( Fig. 2B). In respect to the factor "test", the DC was significantly increased after static stretching $(+3.8 \pm 5.0 \%, \mathrm{p}<0.05)$. For the factor "cycle", the DC decreased during cyclic stretching $(-17.2 \pm 5.2 \%$ for cycle 1 vs. cycle $5, p<0.001$, $\odot$ Fig. 2 B $)$, but the decrease was significant only between cycles 1 and 2 .

\section{Energy stored and energy restituted}

As the dissipation coefficient was only decreased across cycles 1 and 2, the analysis of the E and ER was focused upon these cycles. The analysis for $E$ showed significant main effects for "test" ( $p<0.01$ ) and "cycle" ( $p<0.01)$ indicating that it was decreased after static stretching $(-15.8 \pm 8.7 \%$, ○ Fig. $3 \mathrm{~A})$ and the first cyclic repetition $(-12.3 \pm 4.9 \%$, $\odot$ Fig. $3 \mathrm{~B})$. ER was also significantly decreased after static $(-17.0 \pm 9.4 \%, \mathrm{p}<0.01$, - Fig. $3 C)$, and slightly after the first cycle $(-1.7 \pm 1.5 \%, p<0.05$, - Fig. 3D). A decrease in E was observed for all the subjects, 

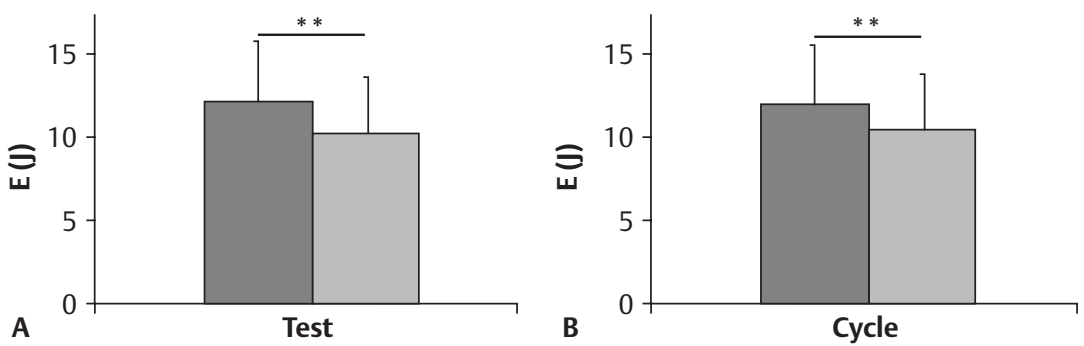

Fig. 3 A to D A Potential elastic energy stored (E) collapsed across the two first cycles before $(\square)$ and after ( $\square$ ) static stretching. B E collapsed before and after static stretching for the first $(\square)$ and the second $(\square)$ cycle. C Potential elastic energy restituted (ER) collapsed across the two first cycles before $(\square)$ and after $(\square)$ static stretching. D ER collapsed before and after static stretching for the first $(\square)$ and the second $(\square)$ cycle. ${ }^{*} p<0.05$, ** $\mathrm{p}<0.01$.
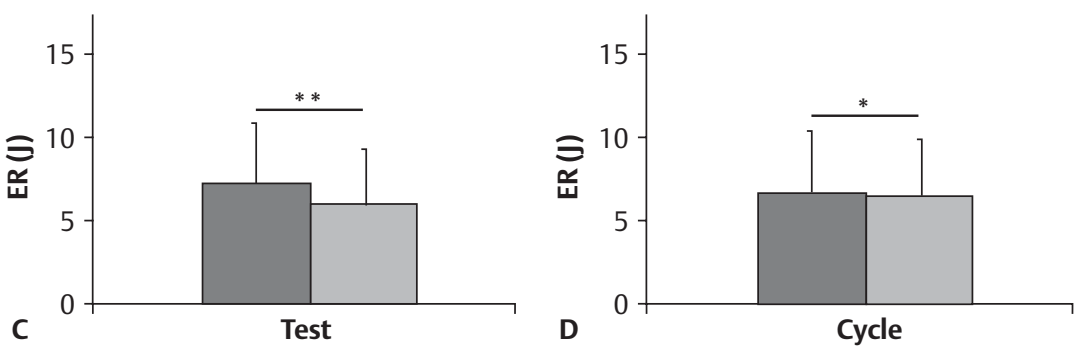

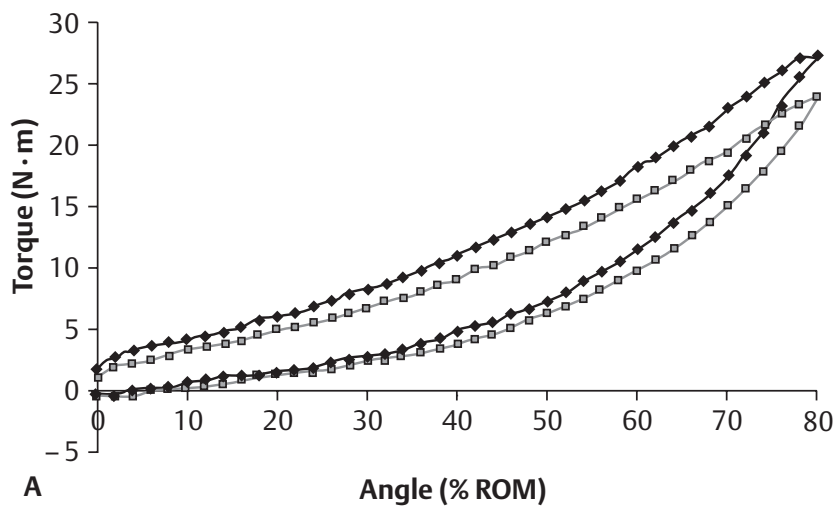

Fig. $4 \mathrm{~A}$ and $\mathrm{B}$ Loading and unloading passive torque-angle relationships to $80 \%$ of the range of motion (ROM) averaged for all the subjects. A Passive

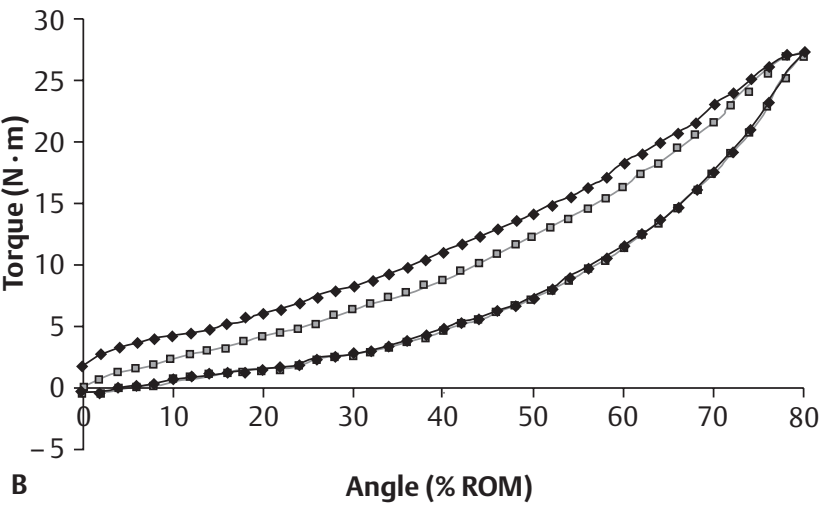

torque for the first cycles before $\bullet$ and after $\square$ static stretching. B Passive torque before static stretching for the first cycle $\bullet$ and the second cycle $\square$. while ER decreased for all the subjects but one. No interaction "test" “ "cycle" was noted for E and ER ( $p>0.05)$.

The torque-angle relationships averaged for all the subjects for trials 1 and 2 at baseline and after static stretching are presented in $\odot$ Fig. 4. It provides an appreciation of how energy changes occurred.

\section{Discussion}

Our results show that the DC and, hence, viscosity was decreased during cyclic stretching. Thus, the shock absorbing ability of this musculo-articular system, primarily hamstring muscles, would be decreased. It was apparent that the decrease occurred over a single cycle of motion, and repeated cycles had little influence (O Fig. 2B). This finding is similar to the results of McNair et al. [14] who reported that the largest changes in musculo-articular elastic properties induced by cyclic stretching occur in the first two repetitions of motion. An original finding of the present study was that following static stretching, the DC was slightly but significantly increased $(+3.8 \%)$. It should be noted on the
- Fig. 2B that DC returned to a similar level to the first trial of the baseline cyclic motion. Consequently, these findings suggest that our static stretching protocol has little influence upon dissipative properties of the system.

The findings related to the DC can be interpreted in relation to energy stored and restituted during the loading and unloading phases of motion ( $\bullet$ Fig. 3). For cyclic stretching, the decrease in energy stored $(-12.2 \%)$ occurred with a decrease in passive torque in the beginning of the ROM ( $\mathrm{Fig} .4 \mathrm{~B})$. Passive torque was minimally affected at the end of the ROM ( $\odot$ Fig. 4B), and the energy restituted was not notably decreased after cyclic stretching $(-1.7 \%$, O Fig. 3D). Subsequently, the ratio between energy restituted and energy stored is then increased, which could explain the decrease in DC after cyclic stretching (equation 1, O Fig. 2 B). In contrast, after static stretching, the decrease in passive torque was more notable at the more extended angles ( $\odot$ Fig. 4A) and both energy stored (-15.7\%) and restituted $(-16.9 \%)$ were decreased ( $\mathbf{O}$ Fig. $\mathbf{3} \mathbf{A}$ and $\mathbf{B}$ ). Magnusson et al. [11] also noted that after static stretching, stored energy was decreased in the middle and the end of the ROM only. 
While the mechanisms associated with the decrease in passive torque and DC following cyclic stretching cannot be determined from the current study, the decrease in the DC could be interpreted as a decrease in viscosity, indicating that the musculo-articular system displays a thixotropic behavior. Indeed, thixotropy is defined as a reversible decrease in viscosity during loading induced by reversible structural changes $[2,12,14,16]$. In the literature, muscle-tendon thixotropy has been explained by three physiological phenomena. Firstly, it has been shown that bonds between actin and myosin filament contributes to the muscle passive tension. Some authors have then argued, using shortrange experiments in isolated muscle, that these bonds are broken by increasing muscle length [17,21]. This phenomenon would then, at least partially, explain decreases in the hysteresis area and the passive musculo-articular tension. Secondly, McNair et al. [14] have proposed that changes in structural arrangement of muscle could possibly occur during motion and induce muscle thixotropy. For instance, the more mobile constituents such as the polysaccharides and water might be redistributed during cyclic stretching. Finally, Kubo et al. [8] have shown that a stretching protocol induces decreases in tendon stiffness and that the hysteresis area of the stress-strain relationship is decreased. Tendons may exhibit thixotropic behavior through the rearrangement/slipping of collagen fibers during stretching [19].

In our study, changes in dissipative properties following stretching protocols were assessed using the DC. In the literature, it has been shown that it is possible to determine muscle or musculoarticular viscosity by imposing small dynamic oscillations and calculating the position-to-torque difference in phase $[3,4,13]$. However, if dynamic perturbation tests had been performed in our study, they would have induced musculo-articular adaptations that might have interfered with the stretching protocols being examined. Hence, the need to examine the DC calculated from the area of hysteresis loop and the stored energy during cyclic stretching. While a main objective of the study was to assess the effects of static stretching, it was also of interest to examine the changes that occurred in the DC across repetitions of cyclic motion. Therefore, in some instances, the cyclic stretching protocols might be viewed as both an intervention and also a dependant variable. With the current design, this could not be avoided. The measurement of energy absorbed and restituted (and thus viscosity) has allowed a deeper appreciation of the mechanisms that might be active when stretching is undertaken. However, the time frame of the changes in dissipative properties induced by stretching has not been studied. It is necessary to assess this time frame to make conclusions about the value of stretching protocols, for instance, in preparation for sport. Using a similar methodology to the current study, Magnusson et al. [11] have shown that despite the immediate effects of 10 cyclic stretching repetitions, there were not significant changes in biomechanical properties ten minutes later. It may be that the time frame of the changes in DC observed in the present study are also of a temporary nature. Further research is needed to address this concern. In summary, the current study examined the dissipation coefficient during cyclic stretching and after static stretching. This coefficient was significantly decreased by cyclic stretching, and was most affected by the first cycle of motion. Changes after cyclic stretching are discussed in terms of musculo-articular thixotropy and three physiological phenomena are proposed to ex- plain thixotropy. Static stretching had little effect upon the damping coefficient, indicating that additional mechanisms have to be considered to explain the acute decrease in passive torque and energy stored following a static stretching protocol.

\section{References}

1 Aagaard P, Simonsen EB, Trolle M, Bangsbo J, Klausen K. Isokinetic hamstring/quadriceps strength ratio: influence from joint angular velocity, gravity correction and contraction mode. Acta Physiol Scand 1995; 154: $421-427$

2 Barnes HA. Thixotropy - a review. J Non-Newtonian Fluid Mech 1997; 70: $1-33$

3 Cornu C, Goubel F, Fardeau M. Muscle and joint elastic properties during elbow flexion in Duchenne muscular dystrophy. J Physiol 2001; 533: $605-616$

4 Desplantez A, Cornu C, Goubel F. Viscous properties of human muscle during contraction. J Biomech 1999; 32: 555-562

5 Esteki A, Mansour JM. An experimentally based nonlinear viscoelastic model of passive joint moment. J Biomech 1996; 29: $443-450$

6 Gajdosik RL. Passive extensibility of skeletal muscle: review of the literature with clinical implications. Clin Biomech 2001; 16: 87-101

7 Hermens HJ, Freriks B, Disselhorst-Klug C, Rau G. Development of recommendations for SEMG sensors and sensor placement procedures. J Electromyogr Kinesiol 2000; 10: 361 - 374

8 Kubo K, Kanehisa H, Kawakami Y, Fukunaga T. Influence of static stretching on viscoelastic properties of human tendon structures in vivo. J Appl Physiol 2001; 90: 520-527

9 Magnusson SP, Simonsen EB, Aagaard P, Kjaer M. Biomechanical responses to repeated stretches in human hamstring muscle in vivo. Am J Sports Med 1996; 24: 622-628

10 Magnusson SP. Passive properties of human skeletal muscle during stretch maneuvers. A review. Scand J Med Sci Sports 1998; 8: 65-77

11 Magnusson SP, Aagard P, Simonsen E, Bojsen-Moller F. A biomechanical evaluation of cyclic and static stretch in human skeletal muscle. Int J Sports Med 1998; 19: 310-316

12 Mandel J. Aperçu sur les principaux comportements rhélogiques. In: Persoz B (ed). La Rhéololgie. Paris: Masson \& Cie, 1969: 1 - 17

13 McFaull SR, Lamontagne A. In vivo measurement of the passive viscoelastic properties of the human knee joint. Hum Mov Sci 1998; 17: $139-165$

14 McNair PJ, Dombroski EW, Hewson DJ, Stanley SN. Stretching at the ankle joint: viscoelastic responses to holds and continuous passive motion. Med Sci Sports Exerc 2001; 33: 354-358

15 McNair PJ, Hewson DJ, Dombroski E, Stanley SN. Stiffness and passive peak force changes at the ankle joint: the effect of different joint angular velocities. Clin Biomech (Bristol, Avon) 2002; 17: 536- 540

16 Persoz B (ed). Introduction à l'étude de la rhéologie. Paris: Dunod, 1960

17 Proske U, Morgan DL. Do cross-bridges contribute to the tension during stretch of passive muscle? J Muscle Res Cell Motil 1999; 20: $433-444$

18 Riemann BL, DeMont RG, Ryu K, Lephart SM. The effects of sex, joint angle, and the gastrocnemius muscle on passive ankle joint complex stiffness. J Athl Train 2001; 36: 369-375

19 Stromberg DD, Wiederielm CA. Viscoelastic description of a collagenous tissue in simple elongation. J Appl Physiol 1969; 26: 857-862

20 Taylor CD, Dalton JD, Seaber AV, Garrett WE. Viscoelastic properties of muscle-tendon units. The biomechanical effetcts of stretching. Am J Sports Med 1990; 18: 300-309

21 Whitehead NP, Gregory JE, Morgan DL, Proske U. Passive mechanical properties of the medial gastrocnemius muscle of the cat. J Physiol 2001; 536: 893-903 